\title{
Bemerkungen zu den Ausführungen von Karl Birnbaum über die Strukturanalyse als klinisches Forschungsprinzip.
}

\author{
Von \\ Artur Kronfeld (Berlin). \\ (Eingegangen am 22. November 1919.)
}

Birnbaum, und ähnlich wie er Schilder und Kretschmer, haben unlängst in einer Reihe ausgezeichneter Arbeiten ernstlich den Versuch gemacht, der Äußerlichkeit klinisch-nosologischer Ordnungen in unserer Wissenschaft das Fundament von Bestimmungen zu geben, die auf die inneren Gründe und Notwendigkeiten des klinischen Geschehens eingehen. $\mathrm{Zu}$ diesen Bestrebungen seien einige kurze und beinahe triviale Bemerkungen gestattet.

Die Klinik, wie sie durch Kraepelin zur Höhe geführt ist, stockt gegenwärtig in der Ertragfülle ihrer Arbeit. Mehr noch - die diagnostischen Auffassungen klinischer Einzelfälle in so manchen Gebieten werden schwankend und widerspruchsvoll. Es bilden sich schulmäßige Gegensätzlichkeiten, und die klinische Forschung selber liefert uns kein Mittel, um sie zu überwinden. Methoden werden herangezogen, die ihrer ganzen Artung nach Ordnungsgesichtspunkte hervorbringen, welche sich ihrerseits mit den klinischen Abgrenzungen nicht decken, ja prinzipiell nichts mit ihnen gemein zu haben brauchen: es sei an die Alzheimerschen Abbaubefunde, an die Abderhaldenschen serologischen Methoden, an die Liquoruntersuchungen Kafkas und der Hamburger Forsehung erinnert. Daß die Klinik von diesen ihr heterologen Methoden, so überaus wertvoll sie als Forschungs-

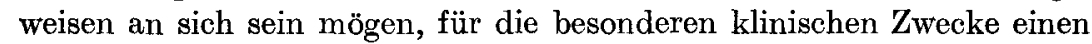
Fortschritt zu erwarten habe, beruht auf grundsätzlich irrigen Hoffnungen. Klinische Fortschritte sind nur von solchen Verfahrensweisen zu erwarten, denen die gleichen teleologischen und theoretischen Gesichtspunkte zugrunde liegen wie der klinischen Nosologie selber.

Damit ist ausgesprochen, wo der Grund für diese Unstimmigkeiten der klinischen Forschung zu suchen ist: darin, daß ihr bestimmte fundamentale und theoretische Gesichtspunkte de facto als oberste Maximen zugrunde liegen, und daß die Klinik sich über diese Maximen keine Rechenschaft gibt 
Birnbaum hat völlig recht; nicht die Verfeinerung der technischen Arbeitshilfen, sondern allein die klare Rechenschaft über den Grundsatzcharakter klinischer Forschungsarbeit überhaupt kann hier weiterhelfen.

Die Klinik sucht Krankheiten, sie sucht Einheiten des kranken Geschehens, und sie schließt auf das Vorliegen derartiger Einheiten aus der Gleichartigkeit oder Ähnlichkeit von Zustandsbildern und Verläufen. Indes ist dies nicht nur, wie die gegenwärtigen Unstimmigkeiten zeigen, ein im Erfolge zweifelhaftes Verfahren; es ist auch ein allzu äußerliches, klarer Gesichtspunkte ermangelndes. Einmal bleibt, was so als ,gleichartig“ oder ,ähnlich" herausgegriffen wird, dem Zufall und der Willkür des Beobachters überlassen. Es mangelt jedes Kriterium dafür, das auf diese Weise Herausgegriffene mit Recht als nosologisch wichtig oder bezeichnend, als symptomatisch zu betrachten. Worin könnte ein solches Kriterium liegen? Doch nur darin, daß das herausgegriffene einzelne als von allgemeiner Bedeutsamkeit für die dahinter supponierte Krankheitseinheit elfaßbar wird, da $B$ es in ihr seinen Realgr und hat, daß es unter ihrem Gesetz steht und aus diesem hervorgeht. Für dieses Hervorgehen gibt es nun verschiedene Stufen und Arten, je nach den akzessorischen Bedingungen, unter denen es aktuell werden könnte. Hiernach gibt es ein System derWertigkeit von Symptomen für eine supponierte Krankheitseinheit - mindestens als Postulat. - Ferner aber fragt die Klinik nicht nach dem Grund der Gleichartigkeiten, nicht nach der Art der Ähnlichkeiten, die sie wahllos und willkürlich zusammenträgt. In beiden Fragen liegt wiederum der Hinweis darauf, daß irgendeine Determination, irgend ein Bestimmungsgrund für alle derartigen Gleichartigkeiten zugrunde liegen muß - oder diese Gleichartigkeiten sind ein nichtssagender Zufall. Und dieser Bestimmungsgrund kann seinerseits wieder nur hergeleitet werden a us de $m$ We sen der Krankheit selber und der in ihrer Realisierung am Einzelfall zusammentreffenden Bedingungen.

Letzten Endes münden alle derartigen prinzipielle Fragen ein in das große Problem der Beziehungen zwischen Krankheits wesen und Krankheitserscheinungen. Und hierbei werden diese Beziehungen gedacht als die systematische Einheit einer ganzen Reihe von gesetzmäßigen Verwobenheiten, eine Einheit, welche eben das „We sen“ der betreffenden Krankheit ausmacht. Der Begriff der Krankheitseinheit ist nur berechtigt als der Begriff eines echten Naturgesetzes: als bloße Konvention, als Arbeitsgesichtspunkt oder als eine sonstige derartige Paraphrase des Relativismus ist er belanglos. In eine derartige systematische Einheit von Gesetzescharakter geht freilich, wie schon gesagt, eine ganze Reihe von Verwobenheiten mit ein, die auf jeweils bestimmte Bedingungen für 
den Realfall der betreffenden Erkrankung zurückgeführt werden. Diese Bedingungen, die man allzu schematisch bisher als „exogene" und ,endogene", individuelle und allgemein-prozeßhafte, dispositionelle und situative, präformierende und auslösende - und was dergleichen Gegensatzpaare mehr sind - geschieden hat, stellen auch ihrerseits gesetzmäßig wirksame Faktoren dar, deren Dignität im Einzelfalle verschieden jst, die aber nicht bloß äußerlich gegen den pathogenetischen Faktor alogewogen werden müssen.

Welches ist der Weg vom äußeren Material der Klinik einer Krankheit zum inneren Gesetz, welches diese Krankheit beherrscht? Schon bei Stellung dieser Frage wird es auffallen, daß der Terminus Krankheit hier ein doppeldeutiger ist. Für die nosologische Klinik bedeutet der Terminus Krankheit nichts anderes als die Summe der herausgehobenen Gleichheits- und Ähnlichkeitsmomente in Zustandsbildern und Verlaufsformen, die gleichsam zum Inhalte eines Begriffs werden, der an sich problematisch bleibt. Im zweiten Falle bedeutet der Terminus Krankheit e in gültiges Gesetz für ein Naturgeschehen; und aus diesem Gesetz soll das Geschehen bestimmbar und herleitbar sein - wenigstens ist dies der grundsätzliche Anspruch; eine andere Frage ist es, wieweit er sich im realen Einzelfalle verwirklichen läßt. Wenn wir also unsere Frage wieder aufnehmen, so wird es uns leicht sein, sie dahin zu beantworten: der Weg von der Krankheit im ersten, klinischen Sinne, zu der Krankheit im zweiten Sinne, ist kein anderer als der Weg von der Erscheinung zum Gesetz.

Wir suchen also das innere Gesetz des Symptomatischen an psychischen $\mathrm{Phänomenen.} \mathrm{Dies} \mathrm{kann} \mathrm{nur} \mathrm{gefunden} \mathrm{werden}$ durch eine immanente Theorie der Symptome. Und man versteht nun leicht, welch neue und gewichtige Rolle der psychopathologischen Symptomatik für den Fortgang der Klinik zuzufallen hat. Ich möchte diesen Gesichtspunkt deshalb besonders hervorheben, weil er sich in Birnbaums vortrefflichen Bemerkungen neben seinen sonstigen Arbeitsgesichtspunkten nicht so betont vorfindet, wie die anderen "strukturanalytischen" Maximen. Er ist aber die grundlegende und entscheidende Vorstufe auch für diese.

Der gewöhnliche Einwand - und ein vollauf berechtigter - hierwider ist der, daß es doch schon, von Griesinger bis zu Wernicke, eine symptomatologische Ära in unserer Wissenschaft gegeben habe, und man wisse, was dabei herausgekommen sei. Konstruktive Hypothesen über einzeln'e herausgelöste Symptome oder Symptomgruppen sind billig; mögen sie nun in hirnpathologischen Theoremen gründen oder irgendeine übergreifende psychologische Grundlehre, wie etwa die assoziative, anwenden. Wirklicher Fortschritt kam erst durch die Inauguration klinisch-nosologischer Gesichtspunkte. 
Wir schließen uns dem Gehalt dieser Meinung durchaus an, an dem Ziel und Plan der immanent-phänomenologischen Symptomatik aber, die wir hier als weiterführenden Weg befürworten, findet jener Einwand keinen Angriffspunkt. Wir sind ja, gerade im Hinblick auf diesen inneren Bauplan der geforderten Symptomatik, gegen jene überlebte, konstruktive Hypothetik, die sich am einzelnen, ohne alle Rechtsbeglaubigung herausgelösten Symptom auslebt. Wir negieren die klinischnosologische Einstellung nicht, sondern wir setzen sie voraus; und unser ganzes Beginnen wäre ohne diese Voraussetzung belanglos. Ist es doch gerade das letzte Ziel der von uns geforderten Arbeitsweise, die zufällig-heuristischen Ontologien der Klinik in eine gesetzmäBig bewegte Dynamik des psychopathologischen Geschehens überzuführen. Freilich müssen sich alsdann an den Bestimmungen dieser Dynaimik, wenn sie vollzogen worden sind, die Abgrenzungen der Klinik im einzelnen korrigieren; der Weg zu dieser Korrektur kann aber nur schrittweise und vorsichtig betreten werden. Wir stimmen daher Körtke nicht bei, wenn er die klinische Ontologie unter Hinzunahme der somatischen Begleitphänomene auf die pathogenetische Einheit des Morbus zuspitzen will und hiervon die - im weitesten Sinne - psychopathologische Betrachtungsweise durch eine unüberbrückbare Kluft abtrennt. Auch die letztere geht in ihren Endabsichten auf die Statuierung dynamisch-genetischer Theorie, und da sind wir der Meinung, daß es nicht zwei verschiedene dynamische Erklärungen für das gleiche Geschehen geben kann, die beide allgemeingültig und gleich richtig sein sollen. Jeder der beiden Wege liefert vielmehr nur eine $\mathrm{n}$ Teil der Bedingungen, die das Geschehen regeln, für jeden von beiden ist die Aufgabe unvollendbar, und a us beiden gemeinsam erwächst die letzte pathogenetische Synthese.

Die immanente Theoretik des psychopathologischen Geschehens, nach seinem phänomenalen Bestande, nach seinen dynamischen Verknüpfungen, nach seinen fundierenden Funktionen, nach deren dispositioneller Bereitschaft und den Ordnungsweisen dieser Bereitschaft, nach ihren konstitutionellen Ausbildungs- und Entwicklungsmöglichkeiten, ferner nach der Auslösbarkeit dieser funktionalen· Kräfte im Seelischen und nach den adäquaten Bedingungen der Auslösung - : die immanente Theoretik nach allen diesen und einer großen Zahl von anderen Gesichtspunkten bedarf aber, um möglich zu werden, einer sehr exakten kritischen und logischen Vorarbeit. Und wir können nicht sagen, daß diese Vorarbeit bis jetzt genügend geleistet worden ist. Was nützt uns der geniale Wurf einer psychiatrischen Charakterologie, wie sie Kretschmer im ,sensitiven Beziehungswahn“ zum Ausgangspunkt seiner Einzelforschungen nimmt, wenn diese Charakterologie logisch und theoretisch noch unbeglaubigt ist; wenn keinerlei immanent- 
psychologische und logische Beziehungsbrücken bestehen zwischen seinen Reaktionsformen (die selber, bei aller intuitiven Sicherheit ihrer Setzung, irgendwie zufällig nach Zahl und Art sind), seinen charakterologischen Typen - und andererseits der konstitutionellen Basis derselben, und wenn bei dieser hinwiederum keine Beziehung aufweisbar ist zu den funktionspsychologischen Klassen und den allgemeineren Bestimmungen psychischer Kausalität? Gerade die außerordentliche zukünftige Bedeutsamkeit der charakterologisehen Versuche Kretschmers und der logischen Rahmengebungen Birnbaums weist uns gebieterisch darauf hin, diese Dinge nicht wie zufällige und willkürliche Inventionen stehen zu lassen, sondern aus einer allgemeineren Theorie heraus zu fundieren. Die Vorarbeit hierzu muß aber phänomenologischer und psychologisch-theoretischer Art sein, und es ist nur vor der Verwechslung dieser Theorie mit den früheren konstruktiven Hypothesen, welche die Psychopathologie von der Hirnphysiologie übernehmen zu müssen glaubte, zu warnen.

Wir sagen also damit, daß derjenige Weg für eine künftige Weiterarbeit der unsrige sein wird, welchen zuerst Jaspers beschritten hat. Das psychische Geschehen ist seinem phänomenalen Wesen nach in allen jenen Beziehungen zu würdigen, in welchen es zur ganzen Persönlichkeit des Subjektes steht, an dem es sich abspielt. Die Erlebnisseite des psychischen Geschehens für sein Subjekt ist hier zweifellos die erste und wichtigste. Eine deskriptive Ontologie der Weisen und Strukturen von Erlebnissen ist zu intendieren. Sie darf aber nicht in ein systemloses analytisches Chaos ausarten, in dem sich Weg und Blick verlieren. Keinen Augenblick dürfen wir vergessen, daß es sich um die synthetische Einheit einer Psyche, eines Subjekts handelt, das wir entweder nur als ein System von Gesetzmäßigkeiten oder gar nicht zu einer wahrhaft wissenschaftlichen Darstellung bringen können. Zur Ermöglichung dieser wissenschaftlichen Darstellung haben wir einen theoretischen Rahmen für diese Gesetzmäßigkeit zu unterlegen, in dem die Einheit der Persönlichkeit als ein Ganzes synthetisch hervorgeht aus einer Reihe funktionaler Bedingungen, welche die psychischen Kräfte für alles in ihr geschehende einzelne abgeben. Diesen Weg habe ich im ersten Bande meiner allgemeinen Psychiatrie, „Das Wesen der psychiatrischen Erkenntnis", $\mathrm{zu}$ beschreiben versucht. Ich habe dort auch $\operatorname{schon}^{1}$ ) die primitivsten psychiatrischen Folgerungen gezogen. Sie decken sich mit der Trennung von Jaspers in solche Phänomene psychotischer Art, die wir als Entwicklung oder Reaktion von Persönlichkeiten - und in solche, die wir als prozeßbedingt bezeichnen. Nämlich aller phänomenale Bestand eines „kranken“, seelischen Geschehens ist - ohne weitere

1) S. 396-421. Springer 1919. 
Bestimmung dessen was mit ,,krank" gemeint ist — entweder aus den gleichen funktionalen Fundamenten hervorgegangen, in denen das Ganze der Persönlichkeit gründet, oder nicht. Diese Disjunktion ist vollständig. Im letzteren Fall nun liegen letztlich immer irgendwie Weisen einer neuartigen funktionalen, intentionalen Wesenheit seelischer Akte zugrunde, die der Persönlichkeit, so wie wir sie als funktionale Einheit begriffen hatten, ,fremd“ sind; sie sind ,,prozeßbe-

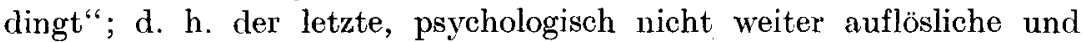
somit ,unmittelbare" Ausdruck eines transpsychischen pathogenen Faktors. Die phänomenologische Analyse des Erlebens, deren Abstraktionsgesichtspunkte aus dieser Disjunktion stammen, führt also mit Eindeutigkeit und Notwendigkeit zur Auffindung immanenter Kriterien für das Vorliegen eines. Prozesses im obigen Sinne, zu seinen Primärsymptomen. Ob ,organischer", ob ,schizophrener", ob irgendwie "funktionaler" Prozeß (wenn man die letztere Begriffsbildung überhaupt geiten lassen will): dies ist vorerst gleichgültig und mit den Mitteln dieser Analyse auch nicht lösbar. Ziehen wir aber hier einmal die überkommenen klinischen Frrungenschaften heran, so wissen wir, daß der praktische Wert dieser Analyse sich so gut wie ausschließlich in der Abgrenzung ,paranoider" und "schizophrener" Prozeße im weitesten Sinne gegenüber den ,reaktiven ", phasenhaften, ,ausgelösten" und situativen psychotischen Bildern dartun muß. Gerade in dieser Abgrenzung liegt aber das große Problemkonvolut der gegenwärtigen Psychiatrie. Da erwächst also der immanent phänomenologischen Aralyse des psychischen Zustandsbildes die Möglichkeit, eine direkte, eindeutige und sichere diagnostische Bestimmung zu treffen. Freilich muß diese Analyse, unter Ausschaltung aller kon* struktiven Vereinfachungen, die Phänomenalität des Erlebens in ihrer ganzen Komplexität ergreifen, und die Maximen der Abstraktion müssen dementsprechend solche sein, die in der intentionellen und funktionalen Psychologie gründen. Ich habe bereits einmal für die schizophrenen Wahnbildungen einen derartigen Versuch unternommen ${ }^{1}$ ); in analoger Weise läßt er sich für die übrigen Gebiete des Erlebens und seiner Weisen durchführen. So für die Trugwahrnehmungen, bei welchen man die originären Momente echter primärsymptomatischer Prozeßhalluzinationen im Wahrnehmungsraum durch immanente Analyse von allen übrigen Täuschungen der Rezeptivität abtrennen kann. Stöcker hat, in seiner Theorie der Genese von Halluzinationen, dies Vorliegen von analytisch-primären Faktoren übersehen. Für die sogenannten Gefühle, Bewußtheiten und sonstigen phänomenalen Repräsentationen von Akten des Interesses liegt überhaupt noch keine diesbezügliche Arbeit

1) Das Erleben in einem Fall von katatoner Erregung. Monatsschr. f. Psych. u. Neurol. 1914, Heft 3. 
vor. Für die erlebnishafte Representation des Ich finden sich einige wegweisende Bemerkungen in einer Arbeit $\mathrm{G} r \mathrm{ruhles}$. Im übrigen hoffe ich in einer demnächst erscheinenden Arbeit die immanenten Kriterien dafür dartun zu können, wann Störungen des Aktivitätsbewußtseins prozeßbedingte Primärsymptome darstellen, wann sie nur Formen einer reaktiven oder phasenhaften Persönlichkeitsentwicklung im Sinne der Depersonalisation Österreichs sind. Im allgemeinen ist das Feld für diese phänomenologische Symptomatik der Zukunft noch. völlig offen. Mir liegt an dieser Stelle daran, ihre Einordnung in den Gesamtgang der Forschung und ihre kaum zu überbietende Wichtigkeit auch unter praktischen Gesichtspunkten darzutun.

Neben diese phänomenologische Analyse des psychischen Geschehens muß die wissenschaftliche Fundierung seiner Dynamik treten. Hier sind nun in der Forschung bereits die verheißungsvollsten Ansätze vorhanden, leider aber in systemloser Weise. Die genetische Theorie der Abfolge des psychischen Geschehens, welche als Fundament aller dieser Bestrebungen erfordert wird, jst nur möglich auf Grund exakter allgemeiner Bestimmungen über das Wesen der psychischen Kausalität und der Beziehungen von Reiz und Bedingung auf die psychische Materie. Auch diese Fundamente habe ich in meinem genannten Buche zu legen versucht. Setzen wir sie als gegeben voraus, so zeigen sich folgende Fragestellungen für die praktische Einzelforschung.

Es sind allgemeine Feststellungen darüber erforderlich, in welchen Weisen Erlebnisse auf das Ganze der erlebenden Persönlichkeit zurückwirken und im Sinne einer Modifikation dieser Persönlichkeit wirken. Solche Feststellungen sind insbesondere notwendig für die primärsymptomatischen Prozeßerlebnisse. Wir haben also die Reiz- oder Bedingungswirkungen dieser Erlebnisse zu studieren. Das Korrelat dazu muß uns eine Lehre von den Strukturt y pen der Gesamtpersönlichkeit liefern, je nach dem Aufbau und den Zusammenordnungsweisen ihrer funktionalen Fundamente. Je nachdem diese Zusammenordnungsweisen dispositionell vorgegeben oder im Laufe ihrer Ausbildung zu prävalenten Bereitschaften und Determinanten zugespitzt sind, wird sich eine Konstitutionslehre und eine Charakterologie der psychischen Persönlichkeit, mit ihren Aktions- und Reaktionsweisen und deren Auslösbarkeit entwickeln lassen. Die wertvollste Vorarbeit hierfür liegt bisher in Kretsch mers Andeutungen seines Buches. Nimmt man das objektiv analytische Fundament primärsymptomatischer Modifikationen, wie wir sie bei Prozessen finden, in das Ganze jener Persönlichkeiten mit hinein, so erhalten wir Formen und Arten schizophrener oder paranoider Charaktere, aus denen wir psychotische Zustände sekundärer, 
324 A. Kronfeld: Bemerkungen zu den Ausführungen von Karl Birnbaum usw.

"pathoplastisch" erwirkter Art im Sinne Birnbaums als ausgelöst, reaktiv oder entwickelt zu begreifen vermögen. Endlich werden uns die Forschungen Freuds und Adlers für die erlebnismäßige Inhaltsbestimmung derartiger Zustände - unter Voraussetzung aller bisher genannten übergeordneten Bedingungen - wichtige dynamische Anhaltspunkte zu vermitteln vermögen.

Alle diese Arbeitsweisen gefährden die klinische Nosologie nicht, sie sind vielmehr berufen sie zu stützen und $\mathrm{zu}$ verimmerlichen. Sie setzen an die Stelle des Technizismus und der Routine eine wahrhaft psychologische Verinnerlichung und Vertiefung. An Stelle der heterologischen Eselsbrücken für die Bequemlichkeit äußerer Diagnostik — als welche man erst unlängst wieder nur allzu gerne die A bderhalde nsche Methodik in Anspruch genommen hätte - an Stelle der neurologischen und hirnpathologischen Dogmatik besinnen sie sich a uf das Wesen psychiatrischer Forsehung als autologischer, oder um mit Ziehen zu sprechen autochthoner Wissenschaft.

Viel mehr als dieses Programm können unsere derzeitigen Bestrebungen noch nicht vorweisen. Mit Recht wird die Forderung gestellt werden, es zunächst einmal zu verwirklichen. Aber schon die ersten vorliegenden Versuche dieser Verwirklichung haben in einer unvergleichlichen Weise zur Hebung des klinischen Forschungsniveaus beigetragen. Mehr noch: sie haben die Berufsfreudigkeit eines jeden von uns endlich wieder erhöht, der sich vorher vergeblich die Frage vorlegte: worin liegt der Sondercharakter unserer Disziplin; und ist sie nicht einfach ein besonders stiefmütterlicher und verkümmerter Zweig am Baume der Gesamtmedizin, der in steriler Ertraglosigkeit warten muß, bis ihn aus den Wurzeln anderer medizinischer Disziplinen - serologischer, biochemischer u. dgl. - fremde Säfte ein wenig in der Entfaltung fördern? Wir glauben dagegen erkannt zu haben, daß dem nicht so ist, daß die Psychiatrie ihrem eigenen Wesen nach ïber Methoden und Gesichtspunkte verfügt, die man nur zutage zu fördern braucht, um ihre wissenschaftliche Zukunft zu sichern. 